\title{
Influence des conditions de culture sur la production de capsules chez le cotonnier (Gossypium hirsutum L.) en conditions de culture pluviale au Bénin
}

\author{
Emmanuel Sekloka ${ }^{(1)}$, Jacques Lançon ${ }^{(2)}$, Valérien Amégnikin Zinsou ${ }^{(1)}$, \\ Grégoire Thomas ${ }^{(3)}$ \\ (1) Université de Parakou. Faculté d'Agronomie. Département de Production végétale. BP 123. Parakou (Bénin). \\ E-mail : emmanuel.sekloka@fa-up.bj \\ (2) Cirad c/o ICRAF. 00100 Nairobi (Kenya). \\ ${ }^{(3)}$ Agrocampus Ouest. 65, rue de Saint Brieuc. CS 84215. F-35042 Rennes cedex (France).
}

Reçu le 22 mai 2015, accepté le 15 décembre 2015.

Description du sujet. Cet article décrit l'effet des facteurs «période de semis » et « densité » sur la répartition des capsules parvenues à maturité sur la plante de cotonnier et sur le rendement final, en prenant des variétés contrastées pour leur port et leur précocité.

Objectifs. Cette étude vise à apporter un appui aux programmes de création variétale pour la culture pluviale du coton en Afrique de l'Ouest, en comprenant comment deux facteurs clés de l'itinéraire technique modifient la mise en place de la production.

Méthode. Pour réaliser cette étude, quatre essais ont été conduits sur deux sites au Bénin en 2002 et 2003 avec six variétés, trois densités de culture $\left(42000,125000,167000\right.$ plantes $^{-a^{-1}}$ ) et deux dates de semis (juin, juillet/aout) suivant un dispositif en split-split plot. Nombre de sites fructifères, taux de rétention, production et répartition capsulaire et rendement en coton graine ont été évalués.

Résultats. En semis retardé, la production de capsules est réduite et celles-ci sont concentrées sur les deux premières positions des six premières branches fructifères (BF). Les fortes densités provoquent une diminution du nombre de sites, du taux de rétention et une concentration de plus de $50 \%$ de la production sur les premières positions des quatre premières BF. Comparées aux variétés sélectionnées en Afrique (H279-1 et Irma A1042), les variétés précoces et compactes Mar 88-214 et Chaco 520 ont une proportion de capsules mûres plus élevée dans leurs parties basses (premières positions des premières $\mathrm{BF}$ ), mais leur rendement moyen est en général plus faible.

Conclusions. La sélection pour une rétention basse élevée (RP1-2_BF1-8) peut améliorer le rendement en condition de culture maitrisée et pour les variétés les moins efficientes, mais l'optimum du taux de rétention est fonction de l'itinéraire technique et, plus généralement, des conditions de culture.

Mots-clés. Gossypium hirsutum, rendement, branche fructifère, maturation, fructification, Bénin.

Influence of cultivation conditions on the production of capsules in cotton (Gossypium hirsutum L.) under rainfed conditions in Benin

Description of the subject. This article describes the effects of the factors "planting date" and "density" on mature boll distribution on the cotton plant and on final yield, focusing on contrasting varieties for habit and earliness.

Objectives. This study aims at supporting the rainfed cotton breeding programs in West Africa in their understanding of how two key factors involved in the cropping system modify the establishment of production.

Method. Four trials were conducted at two sites in Benin in 2002 and 2003, with six varieties, three crop densities $(42,000$, $125,000,167,000$ plants $^{\prime} \mathrm{ha}^{-1}$ ) and two planting dates (June, July/August) following a split-split plot design. The number of fruiting sites, the retention rate, boll production and distribution, and seed cotton yield were evaluated.

Results. When sowing was delayed, there was a reduction in production by the cotton plants, and bolls were concentrated on the first two positions of the first six fruiting branches (BF). High plant densities led to a decrease in the number of sites, the retention rate, and a concentration of more than $50 \%$ of production on the first positions of the first four BF. Compared to African-bred varieties (H279-1 and Irma A1042), the early and compact varieties Mar 88-214 and Chaco 520 showed a higher proportion of mature bolls in their lower parts (first positions of first BF), but their average yield was generally lower. 
Conclusions. Breeding for a high retention rate in the lower part of the plant (RP1-2_BF1-8) can improve performance under controlled cropping conditions or for the least efficient varieties, but the optimum retention rate depends on the cropping system and, more generally, cropping conditions.

Keywords. Gossypium hirsutum, yields, fruiting branch, maturation, fruiting, Benin.

\section{INTRODUCTION}

Le cotonnier Gossypium hirsutum L. produit communément deux à trois fois plus de fleurs qu'il ne peut en conduire à maturité et transformer en capsules récoltables. À cause du caractère indéterminé de la plante, l'avortement d'une fleur pour raison physiologique ou parasitaire ne se traduit pas forcément par une perte de rendement : il est souvent compensé par le maintien d'un autre organe fructifère, qui aurait pu avorter (Hearn et al., 1979; Moussa et al., 2003). En revanche, lorsqu'une capsule est perdue, la disparition n'est pas entièrement compensée par le développement de nouvelles capsules ou le grossissement de capsules existantes sur la même branche fruitière (Constable, 1991). Les chutes d'organes fructifères peuvent aussi engendrer un retard de maturité (Ungar et al., 1987 ; Bange et al., 2004) et éloigner le cotonnier de son potentiel de production si la longueur de la saison des pluies et la disponibilité en éléments nutritifs ne sont pas suffisantes. Cette situation est courante dans les agricultures pluviales africaines (Cretenet et al., 2006). La recherche de variétés ou de conditions de culture favorisant une bonne rétention des capsules est de fait un objectif important en recherche cotonnière (Liu et al., 2006). Cependant, tous les nœuds de la plante ne contribuent pas équitablement à la production chez le cotonnier.

La plupart des études s'accordent sur la contribution des branches fructifères (BF) basses au rendement, mais leur nombre et leur position varient en fonction des conditions culturales et environnementales. Jenkins et al. (1990a) ont trouvé que 66 à $75 \%$ du rendement en coton graine est produit par les premières positions de branche fructifère et que les branches fructifères situées aux nœuds 9 à 14 de la tige principale (à partir du cotylédon) produisent l'essentiel du rendement. Pour Constable (1991), les branches fructifères situées aux nœuds 7 à 13 entretiennent le plus grand pourcentage de capsules et produisent les plus grosses capsules chez le cotonnier. Burch et al. (2002) ont trouvé que les premières positions de branches fructifères comptent pour 70 à $75 \%$ du rendement total, les secondes positions pour 20 à $25 \%$ et les positions extrêmes pour moins de $5 \%$. Pour ces auteurs, les BF situées aux nœuds 8 à 12 produisent plus de $75 \%$ du rendement final. Comparés à la variété ancienne et tardive Stoneville 213, les cultivars modernes, généralement précoces, produiraient plus de rendement sur les BF situées aux nœuds 6 à 8 (Jenkins et al., 1990b).
Au Bénin, le durcissement des contraintes climatiques pour la culture pluviale (Tidjani et al., 2012 ; Nouatin et al., 2014) nous a conduit à mener des travaux pour adapter les outils et les stratégies de sélection variétale (Sekloka et al., 2007a ; 2007b ; 2008). À partir de ces résultats, le programme de création variétale conduit par l'Institut National des Recherches Agricoles du Bénin (INRAB) a produit de nouvelles variétés, en cours de vulgarisation (Sekloka et al., 2009 ; Hougni et al., 2014), dont le type morphologique et phénologique reste toutefois proche des variétés traditionnelles, tardives et à grand développement végétatif. Cet article reprend des données des expérimentations antérieures en focalisant sur deux composantes de l'itinéraire technique qui jouent fortement sur la fructification, la rétention capsulaire et le rendement en culture pluviale: la date de semis, qui conditionne la disponibilité en eau de la culture, et la densité de semis, qui conditionne les effets de compétition au sein d'une population cultivée. Pour mieux comprendre ces effets et en tirer des conséquences pratiques pour la conduite de la culture et la sélection variétale, nous avons travaillé sur six génotypes de cotonnier Gossypium hirsutum L. aux morphologies très contrastées.

\section{MATÉRIEL ET MÉTHODES}

Les essais ont été conduits au Bénin en 2002 et 2003, sur deux sites d'expérimentation du Centre de Recherche Agricole Coton et Fibres du Bénin (CRA$\mathrm{CF})$ : à Okpara $\left(2^{\circ} 41^{\prime} \mathrm{E}, 9^{\circ} 18^{\prime} \mathrm{N}, 320 \mathrm{~m}\right.$ d'altitude) au centre de la zone cotonnière et à Cana $\left(2^{\circ} 5^{\prime} \mathrm{E}, 7^{\circ} 6^{\prime} \mathrm{N}\right.$, 89 m d'altitude) au Sud.

Le site d'Okpara a un régime pluviométrique unimodal. En 2002 et en 2003, les pluies ont été abondantes (Tableau 1) et régulières (Figure 1), comme l'indique la courbe d'évolution du nombre de nœuds au-dessus de la dernière fleur blanche en première position de branche fructifère («Nodes Above White Flower », NAWF). Le NAWF est un indicateur de condition de croissance du cotonnier utilisé pour le pilotage de la culture cotonnière. La décroissance régulière de la courbe de NAWF est signe d'une croissance satisfaisante des cotonniers. Toutes allures s'écartant de cette décroissance régulière sont interprétées comme des situations de stress vécu par le cotonnier et pourraient servir à piloter certaines interventions culturales comme l'irrigation, la 
Tableau 1. Date de semis et pluie utile pour les essais conduits à Cana et Okpara - Sowing date and useful rain for the tests conducted at Cana and Okpara.

\begin{tabular}{llll}
\hline Site & Saison & Date de semis & Pluie utile $(\mathrm{mm})^{1}$ \\
\hline Okpara & 2002 & 26 juin & 826 \\
& & 17 juillet & 755 \\
\cline { 2 - 4 } & 2003 & 20 juin & 858 \\
& & 18 juillet & 701 \\
\hline Cana & 2002 & 28 juin & 499 \\
& & 2 aout & 356 \\
\cline { 2 - 4 } & 2003 & 24 juin & 406 \\
& & 24 juillet & 461 \\
\hline
\end{tabular}

${ }^{1}$ Quantité de pluie tombée de 10 jours avant le semis jusqu'à la première récolte - quantity of rain fallen from 10 days before the sowing until the first harvest. fumure, l'application de régulateur de croissance, etc. (Bourland et al., 1992).

Le site de Cana a un régime pluviométrique bimodal. Les pluies ont été plus irrégulières en cours de culture en 2002 et 2003, avec une sècheresse marquée au mois de septembre (Tableau 1, Figure 2). Pour les deux années, les pluies disponibles pour la culture ont été plus faibles à Cana qu'à Okpara.

Les sols, pauvres en matières organiques, étaient de type ferralitique à Cana (très peu fertile) et ferrugineux tropicaux (fertilité meilleure) à Okpara (Tableau 2).

Le dispositif expérimental est un split-split plot à trois répétitions croisant trois facteurs : deux dates de semis en grande parcelle, trois densités de culture en moyenne parcelle et six variétés en petites parcelles.
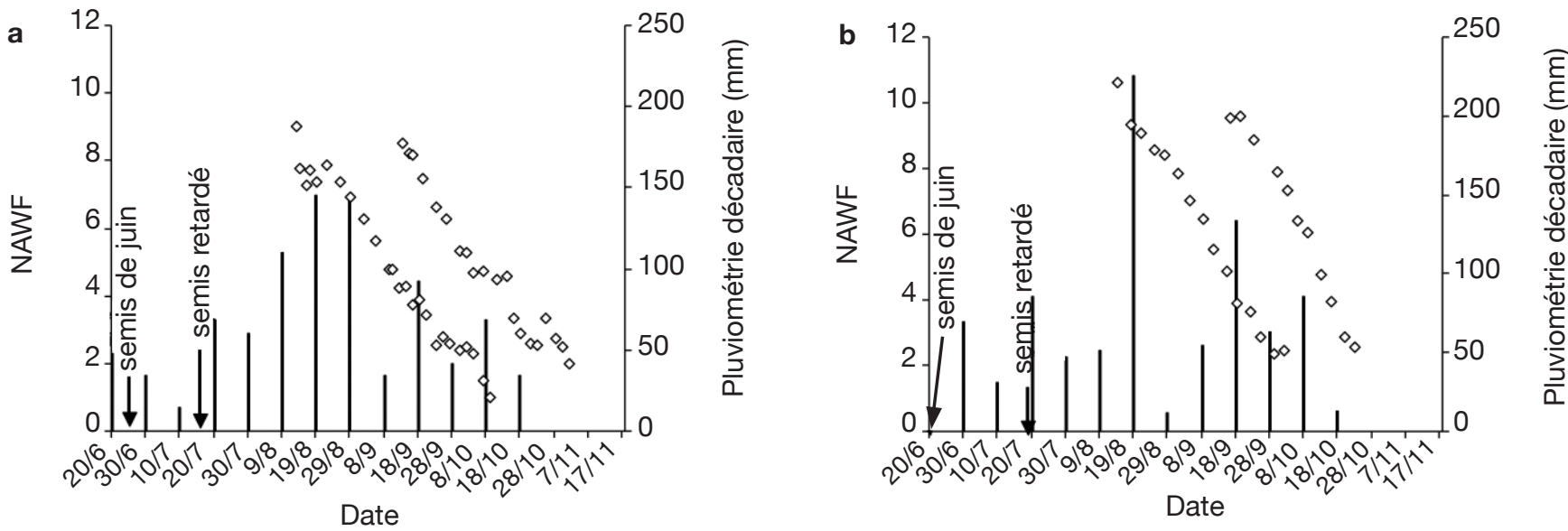

Figure 1. Okpara : pluviométrie et évolution du NAWF $(\diamond)$ en 2002 (a) et 2003 (b) (moyenne des relevés effectués pour six variétés) - Okpara: rainfall and evolution of the NAWF $(\diamond)$ in 2002 (a) and 2003 (b) (average for six varieties).

NAWF : nombre de nœuds au-dessus de la dernière fleur blanche en première position de la branche fructifère - number of knots over the last white flower in first position of the fruiting branch.
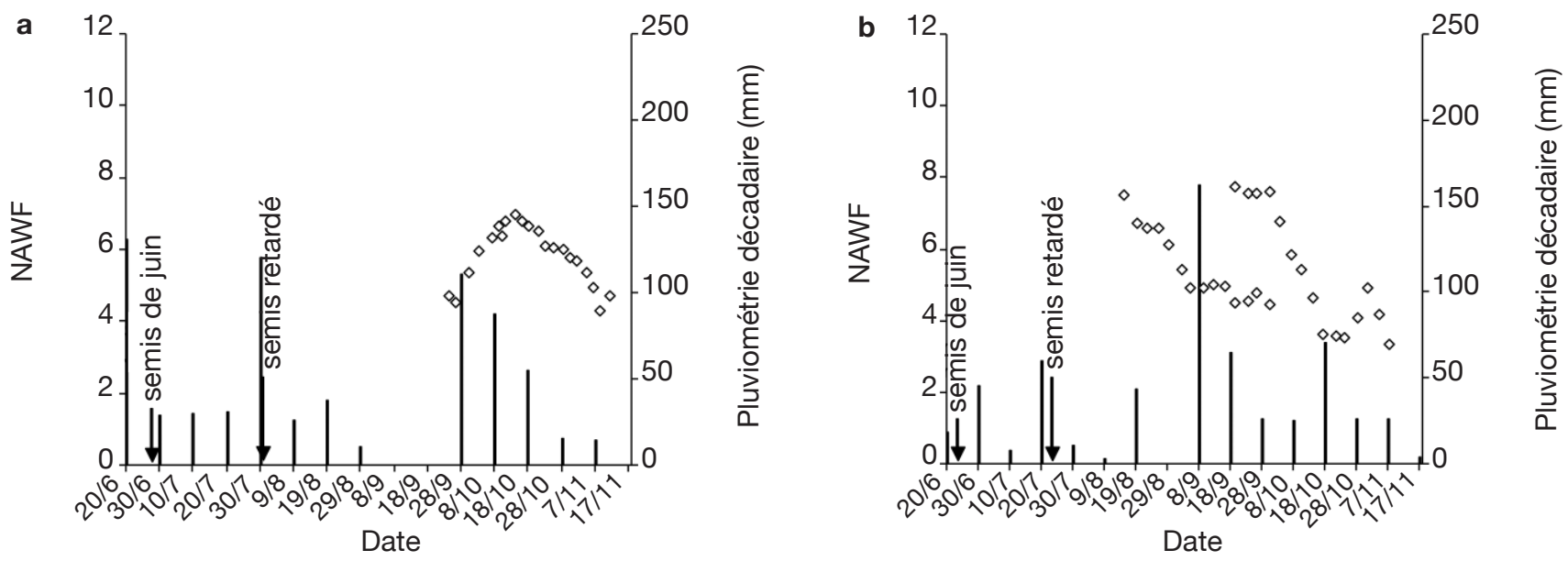

Figure 2. Cana : pluviométrie et évolution du NAWF $(\diamond)$ (voir figure 1) en 2002 (a) et 2003 (b) (moyenne des relevés effectués pour six variétés) - Cana: rainfall and evolution of NAWF $(\diamond)$ (see figure 1) in 2002 (a) and 2003 (b) (average for six varieties). 
Tableau 2. Caractéristiques moyennes des sols de Cana et Okpara pour l'horizon 0-20 cm en 2002 - Mean characteristics of the Okpara and Cana soils in the 0-20 cm horizon in 2002 (Azontondé et al., 2009).

\begin{tabular}{lllllr}
\hline Site & $\mathbf{p H}$ & $\begin{array}{l}\text { Argiles et } \\
\text { limons }(\%)\end{array}$ & $\begin{array}{l}\text { C total } \\
(\%)\end{array}$ & $\begin{array}{l}\mathbf{N} \text { total } \\
(\%)\end{array}$ & $\mathbf{C} / \mathbf{N}$ \\
\hline Cana & 6,2 & 23,50 & 0,94 & 0,078 & 12,05 \\
Okpara & - & 22,40 & 1,43 & 0,167 & 8,56 \\
\hline
\end{tabular}

La première date de semis, correspondant aux premières pluies, a pu être réalisée fin juin. La date « retardée » s'est située entre 3 et 5 semaines après la première.

Les trois densités de culture sont 42000 plantes $^{\prime}$ ha $^{-1}$ (écartement $\quad 0,8 \times 0,3 \mathrm{~m}$ ), 125000 plantes $\cdot \mathrm{ha}^{-1}$ $(0,4 \times 0,2 \mathrm{~m}), 167000$ plantes $^{-1} \mathrm{ha}^{-1}(0,4 \times 0,15 \mathrm{~m})$.

Les six variétés utilisées ont une morphologie et une longueur de cycle très contrastées. La variété réputée la plus précoce est Mar 88-214 et la plus tardive est Irma A1042. La morphologie est très différente entre la variété Mar 88-214, à port compact, à entrenœuds et branches fructifères très courts, et les types conventionnels cultivés en Afrique, comme Stam 18A et Irma A1042, dont le développement végétatif est beaucoup plus vigoureux (Tableau 3).

Les parcelles élémentaires sont constituées de 3 ou 6 lignes de $6 \mathrm{~m}$, selon les densités, de manière à conserver une surface constante de $14,4 \mathrm{~m}^{2}$. Elles sont démariées à une plante par poquet. La fertilisation a été appliquée suivant les recommandations de la vulgarisation: $200 \mathrm{~kg} \cdot \mathrm{ha}^{-1}$ d'engrais complet de formule 14-23-14 au semis et $50 \mathrm{~kg} \cdot \mathrm{ha}^{-1}$ d'urée $(46 \% \mathrm{~N})$ à la floraison seulement pour le semis précoce. La protection phytosanitaire a été renforcée pour limiter les chutes d'organes fructifères liées aux attaques d'insectes. Au total, 10 traitements insecticides hebdomadaires ont été réalisés à partir de l'apparition des premières fleurs jusqu'aux premières récoltes. Les deux premiers traitements étaient à base d'endosulfan $\left(21 \cdot h^{-1}\right)$, les quatre suivant à l'aide d'un binaire acaricide (cyperméthrine associée à chlorpyrifos-éthyl, $11 \cdot \mathrm{ha}^{-1}$ ) et les quatre derniers à base d'un binaire aphicide (cyperméthrine associée au diméthoate, $11 \cdot \mathrm{ha}^{-1}$ ).

Les observations ont été réalisées sur les lignes centrales des parcelles élémentaires ( $2^{\mathrm{e}}$ ligne pour les parcelles de 3 lignes, $3^{\mathrm{e}}$ et $4^{\mathrm{e}}$ lignes pour les parcelles de 6 lignes). Cinq plantes choisies au hasard dans chaque parcelle élémentaire ont été décrites suivant la technique de "plant mapping » (Bourland et al., 1990), en localisant les positions de capsules le long de chaque branche végétative (BV) et de chaque branche fructifère ou sympode (BF) (Figure 3). On a ainsi pu calculer le nombre de sites fructifères émis par plante, la proportion de ces sites fructifères qui ont évolué en capsules récoltables (taux de rétention capsulaire) et le nombre de capsules récoltées à différents endroits au niveau de la plante.

Les analyses de la variance ont été réalisées en utilisant la procédure Mixed du logiciel SAS® (SASInstitute, 1988). Les moyennes ont été comparées à l'aide du test de Tukey-Kramer (1956).

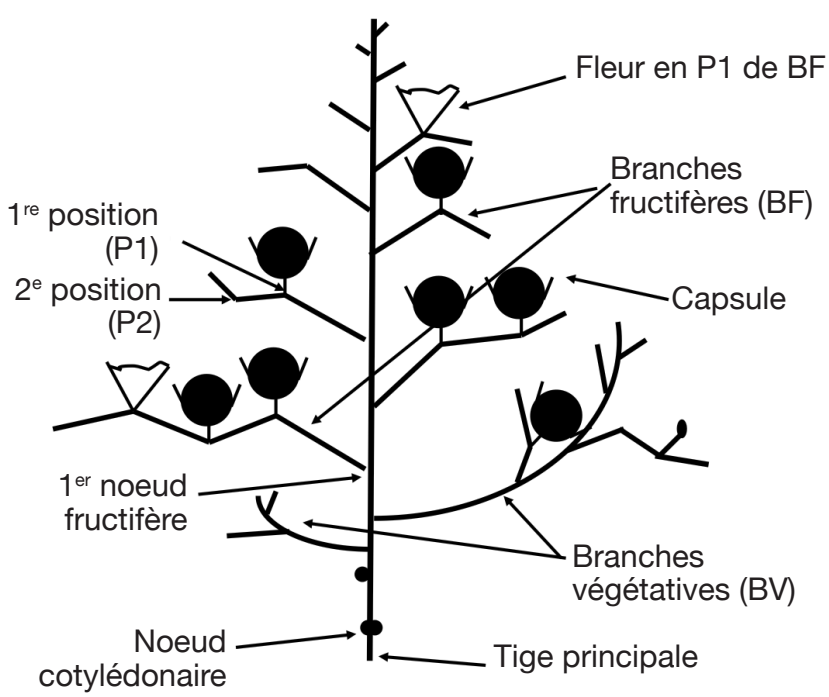

Figure 3. Structure du plant de cotonnier montrant les branches et les nœuds fructifères - Structure of the cotton plant showing branches and fruiting nodes.

Tableau 3. Origine et morphologie des variétés testées - Origin and morphology of the varieties under test.

\begin{tabular}{lllll}
\hline Variété & Origine & Zone de culture & Précocité & Port \\
\hline Mar 88-214 & USA & USA & Précoce & Compact-branches courtes \\
Chaco 520 & Argentine & Argentine & Moyenne & Compact \\
Guazuncho 2 & Argentine & Argentine & Moyenne & Compact \\
H 279-1 & Togo - Bénin & Bénin & Tardive & Élancé-arborescent \\
Irma A 1042 & Cameroun & Cameroun & Tardive & Élancé-arborescent \\
Stam 18 A & Togo - Bénin & Bénin & Tardive & Élancé-arborescent \\
\hline
\end{tabular}




\section{RÉSULTATS}

Globalement, dans les deux sites, on observe une corrélation négative entre le nombre de nœuds fructifères produits et le taux de conversion des organes floraux en capsules ou taux de rétention capsulaire (Figure 4). Cette corrélation négative est plus forte à Cana $(-0,84)$ qu'à Okpara $(-0,28)$ où la pluviométrie était supérieure.

\subsection{Effet de la date de semis}

Taux de rétention global et nombre de sites fructifères. L'effet de la date de semis sur le taux de rétention capsulaire est variable suivant les localités. À Cana, pour chacune des deux années, les taux de rétention ont été meilleurs sur les semis retardés: les cotonniers y ont produit moins de fleurs et en ont converti une proportion plus importante en capsules récoltables. À Okpara, par contre, les taux de rétention ont été un peu plus élevés sur les semis de juin, les semis retardés ayant produit plus de fleurs que ceux de juin (Figure 4).
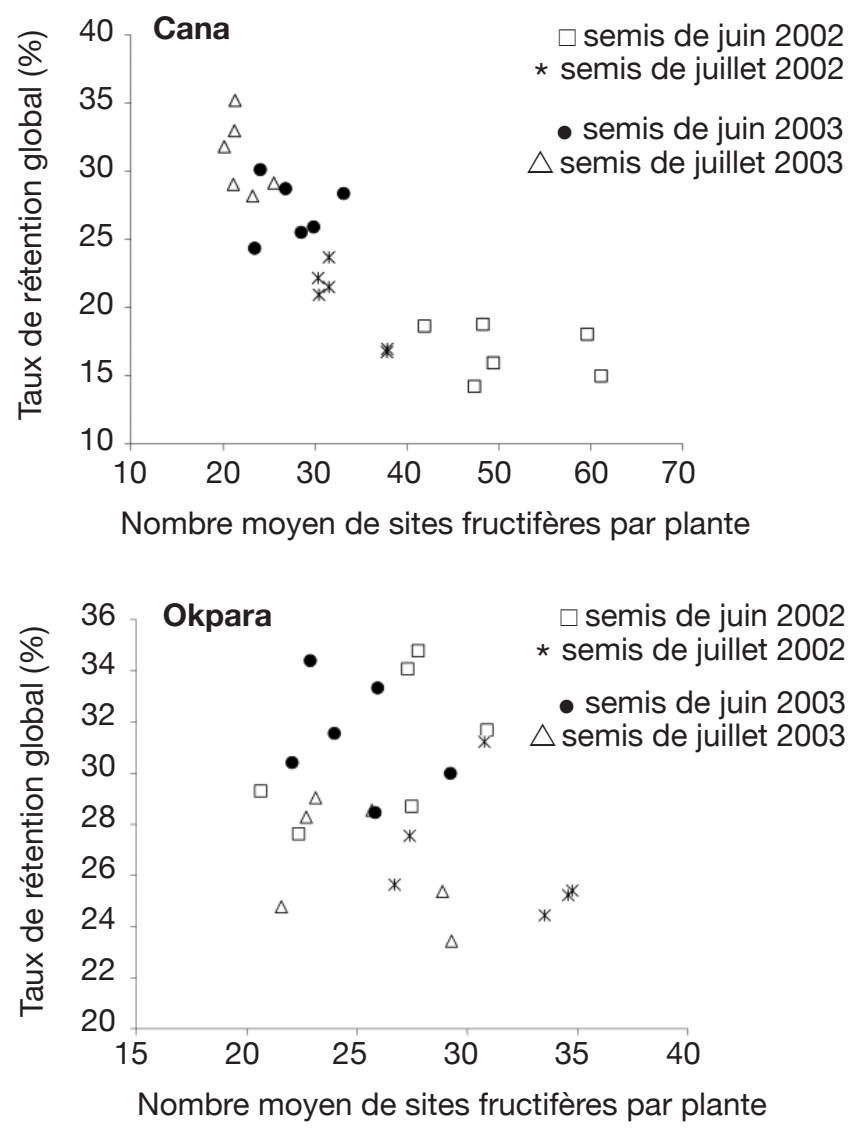

Figure 4. Nombre moyen de sites fructifères par plante aux différentes dates de semis dans chaque lieu - Average number of fruiting sites in function of the different sowing dates in each location.
Production capsulaire et répartition. La date de semis a très peu d'effet sur le nombre moyen de capsules récoltées par plante. Les différences entre dates de semis ne sont pas significatives $(p=0,06)$, même si les nombres de capsules par plante sont en moyenne légèrement plus faibles en semis retardé $(6,4$ contre $7,7, \mathrm{LSD}=1,4$ )

La contribution des branches végétatives $(\mathrm{BV})$ à la production a été faible en général et davantage en semis retardé ; en moyenne par date de semis, le pourcentage de capsules sur BV a été de $8,0 \%$ en semis de juin contre $6,9 \%$ en semis retardé $(p<0,01)$.

Sur les branches fructifères (BF), les deux premiers nœuds portent l'essentiel de la production capsulaire, avec toutefois une concentration capsulaire plus forte sur ces positions en semis retardé. On a dénombré en semis de juillet $70,3 \%$ de capsules en première position de branches fructifères contre $66,6 \%$ à la même position en semis de juin (Tableau 4).

La majorité de la production capsulaire de la plante est située plus bas sur les cotonniers issus de semis retardés : $70 \%$ de la production capsulaire proviennent des deux premiers nœuds des six premières sympodiales, alors qu'ils se répartissent sur les huit premières branches fructifères en cas de semis précoce (Tableau 4).

\subsection{Effet de la densité}

Taux de rétention global et nombre de sites fructifères. À faible densité, les cotonniers sont plus florifères et retiennent mieux leurs capsules. Les nombres moyens de sites fructifères par plante et les taux de rétention sont plus élevés à 42000 plantes. ha $^{-1}$, qu'à 125000 et 167000 plantes $\cdot h^{-1}$ (Figure 5) ;

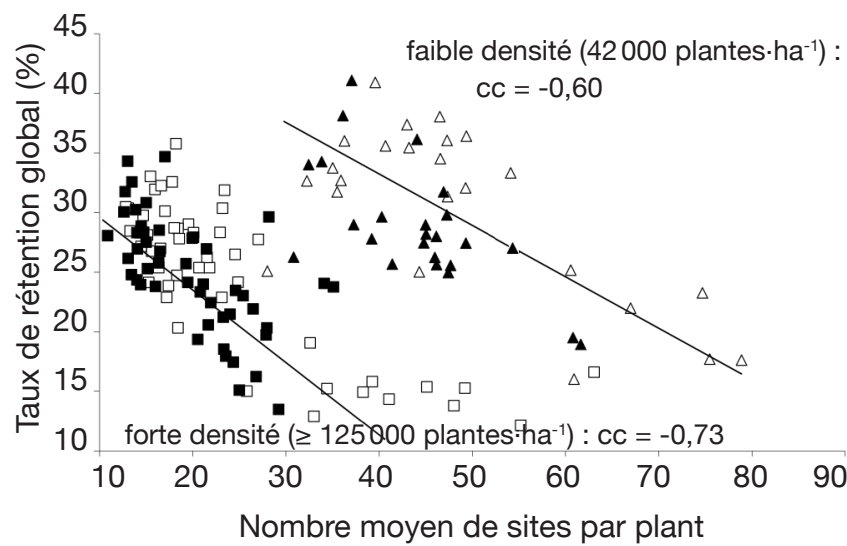

$\Delta$ semis de juin et $\boldsymbol{\Delta}$ semis retardé, 42000 plantes $\cdot$ ha $^{-1}$ $\square$ semis de juin et $\square$ semis retardé, $\geq 125000$ plantes $\cdot$ ha $^{-1}$

Figure 5. Taux de rétention global et relation avec le nombre de sites fructifères - Global retention rate and relation with the number of fruiting sites. 
Tableau 4. Pourcentage de capsules récoltées cumulé par position selon les périodes de semis (pour 1080 plants) - Percentage of harvested bolls cumulated by position for both sowing periods (from 1,080 plants).

\begin{tabular}{|c|c|c|c|c|c|c|c|c|}
\hline \multirow{2}{*}{$\begin{array}{l}\text { Branche } \\
\text { fructifère }\end{array}$} & \multicolumn{4}{|c|}{ Semis de juin } & \multicolumn{4}{|c|}{ Semis retardé } \\
\hline & $\mathbf{P 1}$ & $\mathbf{P 2}$ & P3 & $P \geq 4$ & P1 & $\mathbf{P 2}$ & $\mathbf{P 3}$ & $P \geq 4$ \\
\hline $\mathrm{BF} 1$ & 6,8 & 2,4 & 0,6 & 0,2 & 11,3 & 2,6 & 0,6 & 0,0 \\
\hline BF2 & 14,4 & 5,4 & 1,2 & 0,4 & 22,8 & 5,8 & 1,5 & 0,0 \\
\hline BF3 & 22,8 & 8,2 & 2,1 & 0,7 & 33,2 & 9,0 & 2,4 & 0,0 \\
\hline BF4 & 30,9 & 10,6 & 2,7 & 1,0 & 42,3 & 11,9 & 3,0 & 0,0 \\
\hline BF5 & 38,5 & 12,5 & 3,0 & 1,2 & 50,4 & 14,0 & 3,4 & 0,0 \\
\hline BF6 & 45,9 & 14,0 & 3,2 & 1,5 & 57,2 & 15,5 & 3,6 & 0,0 \\
\hline BF7 & 51,6 & 14,9 & 3,6 & 1,7 & 62,4 & 16,5 & 3,8 & 0,0 \\
\hline BF8 & 56,0 & 15,6 & 3,8 & 1,9 & 66,0 & 17,0 & 3,8 & 0,0 \\
\hline BF9 & 59,0 & 16,2 & 3,9 & 2,1 & 68,2 & 17,3 & 3,9 & 0,0 \\
\hline BF10 & 61,2 & 16,6 & 4,1 & 2,2 & 69,3 & 17,5 & 3,9 & 0,0 \\
\hline $\mathrm{BF} \geq 11$ & 66,6 & 17,8 & 4,4 & 2,3 & 70,3 & 17,7 & 3,9 & 0,0 \\
\hline
\end{tabular}

$\mathrm{BF}$ : branche fructifère - fruiting branch; $\mathrm{P}$ : position de la branche fructifère - position of the fruiting branch; $\mathrm{BF} \geq 11$ : branches fructifères au-delà de 10 - fruiting branches beyond $10 ; \mathrm{P} \geq 4$ : positions au-delà de 3 - positions beyond 3 ; les pourcentages ont été calculés à partir de 1080 plantes pour chacune des dates de semis $(5$ plantes par parcelle $\mathrm{x} 3$ répétitions $\mathrm{x} 6$ variétés $\mathrm{x} 3$ densités $\mathrm{x}$ 2 lieux $\times 2$ années) - the percentages were calculated from 1,080 plants for each sowing date (5 plants per plot $x 3$ repetitions $x$ 6 varieties $\times 3$ densities $\times 2$ places $\times 2$ years).

on peut aussi observer qu'à chaque niveau de densité, les nuages de points correspondant aux semis de juin sont plus dispersés que ceux correspondant aux semis retardés ; les semis retardés réduisent les écarts entre variétés pour le nombre de sites fructifères et le taux de rétention.

En considérant les résultats par groupe de densité, on retrouve une relation négative entre le taux de rétention et le nombre de sites fructifères par plante (Figure 5).

Production et répartition capsulaire. L'augmentation de la densité réduit le nombre moyen de capsules par plante. En moyenne par densité, les nombres moyens de capsules par plante ont été de 11,$4 ; 5,3$ et 4,4 respectivement à 42000,125000 et 167000 plantes. $\operatorname{ha}^{-1}(p<0,01)$.

La contribution des branches végétatives à la production est faible en général, et même négligeable à forte densité $(13,1 \%, 2,9 \%$ et $1,1 \%$ respectivement

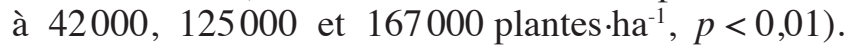
Cette contribution est en outre très aléatoire, comme en témoignent les valeurs élevées des coefficients de variation obtenus à l'issue des analyses de variance (compris entre $27 \%$ et $70 \%$, données non montrées).

Aux fortes densités, les capsules sont davantage concentrées sur les premiers nœuds des branches fructifères basses (Tableau 5). Ainsi, les capsules situées sur les positions 3 et 4 , plus externes, représentent moins de $2 \%$ du total pour les plantes cultivées aux fortes densités contre plus de $8 \%$ à la densité la plus basse. Alors que $50 \%$ du total des capsules sont obtenues sur les $1^{\text {ers }}$ nœuds fructifères des 4 premières sympodiales, il faut 10 sympodiales à la basse densité.

\subsection{Variabilité génétique de la production et de la répartition capsulaire}

En moyenne, les branches végétatives ont très peu contribué à la production, en dépit de différences hautement significatives $(p<0,01)$ entre variétés compactes (Mar 88-214 avec 3\%) et végétatives (Stam 18A avec un peu plus de 6\%, données non montrées). La majorité de la production capsulaire étant localisée au niveau des deux premiers nœuds des premières branches fructifères $(\mathrm{BF})$, le tableau 6 montre que $70 \%$ des récoltes sont obtenues dès la sixième $\mathrm{BF}$ pour Mar 88-214 et Chaco 520, la septième pour Stam 18A et Guazuncho 2, et seulement à la huitième pour Irma A1042 et H 279-1. Chez toutes les variétés, les quatre premières $\mathrm{BF}$ ont porté chacune plus de $10 \%$ de la production capsulaire; les cinq premières $\mathrm{BF}$ réunies ont enregistré plus de $50 \%$ de la production totale de la plante. La production capsulaire devient négligeable au-delà de la $\mathrm{BF} 13$ pour Chaco 520 et Mar 88-214, tandis qu'il faut attendre la BF17 pour Irma A1042, variété très élancée : plus une variété est précoce, plus la proportion de la récolte produite par les premières branches fructifères est importante. 
Tableau 5. Pourcentage de capsules récoltées cumulé par position selon les densités - Percentage of harvested bolls cumulated by position and by densities.

\begin{tabular}{|c|c|c|c|c|c|c|c|c|}
\hline \multirow{2}{*}{$\begin{array}{l}\text { Branche } \\
\text { fructifère }\end{array}$} & \multicolumn{4}{|c|}{42000 plantes $^{\prime} \mathrm{ha}^{-1}$} & \multicolumn{4}{|c|}{$\geq 125000$ plantes $^{\prime} \mathrm{ha}^{-1}$} \\
\hline & $\mathbf{P 1}$ & $\mathbf{P 2}$ & $\mathbf{P 3}$ & $P \geq 4$ & $\mathbf{P 1}$ & $\mathbf{P 2}$ & $\mathbf{P 3}$ & $P \geq 4$ \\
\hline $\mathrm{BF} 1$ & 6,0 & 3,4 & 0,9 & 0,3 & 12,5 & 1,4 & 0,1 & 0,0 \\
\hline $\mathrm{BF} 2$ & 12,2 & 7,5 & 2,2 & 0,7 & 25,9 & 3,2 & 0,3 & 0,1 \\
\hline $\mathrm{BF} 3$ & 18,5 & 11,8 & 3,6 & 1,1 & 39,0 & 4,7 & 0,5 & 0,1 \\
\hline BF4 & 24,8 & 15,3 & 4,6 & 1,4 & 50,3 & 6,2 & 0,7 & 0,2 \\
\hline BF5 & 30,8 & 18,0 & 5,1 & 1,6 & 60,4 & 7,3 & 0,8 & 0,3 \\
\hline BF6 & 36,5 & 20,1 & 5,6 & 1,9 & 69,3 & 8,1 & 0,8 & 0,4 \\
\hline BF7 & 41,4 & 21,4 & 5,9 & 2,0 & 75,3 & 8,6 & 1,0 & 0,5 \\
\hline BF8 & 45,6 & 22,2 & 6,0 & 2,2 & 79,1 & 9,0 & 1,1 & 0,5 \\
\hline BF9 & 48,5 & 22,8 & 6,1 & 2,4 & 81,5 & 9,2 & 1,2 & 0,6 \\
\hline BF10 & 50,4 & 23,2 & 6,3 & 2,4 & 82,8 & 9,4 & 1,2 & 0,6 \\
\hline $\mathrm{BF} \geq 11$ & 53,9 & 24,1 & 6,5 & 2,5 & 86,1 & 10,0 & 1,3 & 0,6 \\
\hline
\end{tabular}

$\mathrm{BF}, \mathrm{P}, \mathrm{BF} \geq 11, \mathrm{P} \geq 4$ : voir tableau 4 - see table 4 ; les pourcentages ont été calculés à partir de 240 plantes pour la densité de 42000 plante $\cdot \mathrm{ha}^{-1}$ ( 5 plantes par parcelle x 3 répétitions x 6 variétés x 1 densité $\mathrm{x} 2$ dates de semis x 2 lieux x 2 années) et 480 plantes pour celle $\geq 125000$ plantes $\cdot \mathrm{ha}^{-1}$ ( 5 plantes par parcelle $\mathrm{x} 3$ répétitions $\mathrm{x} 6$ variétés $\mathrm{x} 2$ densités $\mathrm{x} 2$ dates de semis $\mathrm{x}$ 2 lieux x 2 années) - the percentages were calculated from 240 plants for the density of 42000 plants.ha ${ }^{-1}$ (5 plants per plot $x$ 3 repetitions $x 6$ varieties $x 1$ density $x 2$ sowing dates $x 2$ places $x 2$ years) and 480 plants for the density $\geq 125,000$ plants.ha-1 ( 5 plants per plot $\times 3$ repetitions $\times 6$ varieties $\times 2$ densities $\times 2$ sowing dates $x 2$ places $\times 2$ years).

Dans nos conditions de culture, le taux de conversion des fleurs en capsules récoltables est en moyenne compris entre 38 et $46 \%$ aux deux premières positions (P1-2) des BF1 à 8 (Figure 6). Les quatre variétés les plus productives Guazuncho 2, Irma A1042, H 279-1

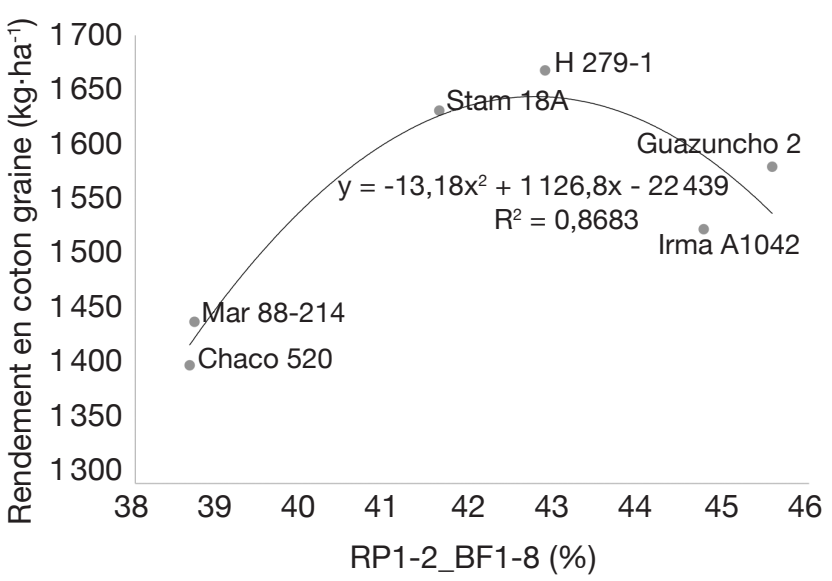

Figure 6. Relation entre le taux de rétention des deux premiers noeuds des huit premières branches fructifères (RP1-2_BF1-8) et le rendement en coton-graine - Relation between the retention rate of the first two nodes of the first eight fruiting branches (RP1-2_BF1-8) and the seed cotton yield. et Stam 18A ont aussi les taux de rétention les plus élevés. Toutefois, les taux de rétention très élevés de Guazuncho 2 et Irma A1042 ne permettent pas d'atteindre d'aussi hauts rendements que les taux plus modérés des variétés locales (sélectionnées au Bénin) H 279-1 et Stam 18A.

Par ailleurs, on observe une corrélation positive forte $\left(\mathrm{R}^{2}: 0,87, p<0,01\right)$ entre le taux de rétention sur les deux premières positions des huit premières branches fructifères et le rendement en coton graine (Figure 6). Cette relation forte se traduit par une régression polynomiale qui admet un optimum à $42,75 \%$ proche des taux de rétention des variétés locales H 279-1 et Stam 18A.

\section{DISCUSSION ET CONCLUSIONS}

\subsection{Effet de la date de semis}

La date de semis joue sur le nombre de sites fructifères et le taux de rétention capsulaire en fonction des apports en eau de pluie au moment de la floraison. Que ce soit à Cana (semis de juin) où à Okpara (semis retardé), les stress hydriques ressentis au moment de la floraison se traduisent par une augmentation du nombre de sites et une diminution du taux de rétention. À Cana, les cotonniers semés en juin 
Tableau 6. Pourcentages de capsules sur les deux premières positions des branches fructifères (BF) des différentes variétés de coton - Percentages of bolls on the first two positions of the fruiting branches $(B F)$ of the different varieties of cottton.

\begin{tabular}{lcccccc}
\hline Branche fructifère & Mar 88-214 & Chaco 520 & Stam 18A & Guazuncho 2 & Irma A1042 & H 279-1 \\
\hline BF1 & 13,31 & 13,32 & 10,56 & 11,83 & 9,89 & 10,36 \\
BF2 & 14,65 & 13,70 & 11,93 & 12,85 & 11,32 & 11,13 \\
BF3 & 14,26 & 13,53 & 11,43 & 12,44 & 11,19 & 11,95 \\
BF4 & 12,79 & 11,28 & 10,81 & 11,04 & 11,08 & 10,46 \\
BF5 & 10,40 & 9,94 & 9,66 & 9,64 & 9,38 & 9,92 \\
BF6 & $\underline{\mathbf{8 , 4 5}}$ & $\underline{\mathbf{8 , 3 1}}$ & 8,75 & 8,54 & 9,15 & 8,22 \\
BF7 & 5,24 & 5,56 & $\underline{\mathbf{7 , 0 5}}$ & $\underline{\mathbf{6 , 5 3}}$ & 7,04 & 6,67 \\
BF8 & 2,64 & 5,39 & 4,82 & $\underline{\mathbf{5 , 4 1}}$ & $\underline{\mathbf{4 , 9 1}}$ \\
BF9 & 1,86 & 3,58 & 3,14 & 3,77 & 3,32 \\
BF10 & 2,51 & 2,57 & 1,78 & 2,21 & 2,61 \\
BF11 & 1,00 & 1,42 & 1,41 & 1,33 & 1,56 & 1,86 \\
BF12 & 1,04 & 1,00 & 0,72 & 0,68 & 0,99 & 1,39 \\
BF13 & 0,65 & $\underline{0,42}$ & 0,61 & 0,89 & 0,48 & 1,12 \\
BF14 & $\underline{0,43}$ & 0,21 & $\underline{0,22}$ & 0,51 & 0,31 & 0,74 \\
BF15 & 0,48 & 0,25 & 0,18 & $\underline{0,24}$ & 0,20 & $\underline{0,47}$ \\
BF16 & 0,48 & 0,08 & 0,25 & 0,14 & 0,07 & 0,10 \\
BF17 & 0,43 & 0,04 & 0,07 & 0,03 & $\underline{0,27}$ & 0,27 \\
$>$ BF18 & 0,13 & 0,00 & 0,11 & 0,03 & 0,06 & 0,23 \\
\hline
\end{tabular}

Les valeurs en gras souligné correspondent au niveau de BF où $70 \%$ des capsules ont été produites et celles en italique souligné correspondent à $99 \%$ des capsules - the values in underlined bold correspond to the level of BF where $70 \%$ of the bolls were produced and the values in underlined italics to $99 \%$ of the bolls; les pourcentages ont été calculés à partir de 360 plantes pour chacune des variétés ( 5 plantes par parcelle $\times 3$ répétitions $\mathrm{x} 1$ variété $\mathrm{x} 3$ densités $\mathrm{x} 2$ dates de semis $\mathrm{x} 2$ lieux $\mathrm{x} 2$ années) - the percentages were calculated from 360 plants for each variety (5 plants per plot $\times 3$ repetitions $x 1$ variety $x 3$ densities $\times 2$ sowing dates $x 2$ places $x$ 2 years).

fleurissent de la mi-aout à la fin septembre, période qui coïncide avec une petite sècheresse habituelle au sud du pays. L'irrégularité des pluies induit une chute élevée d'organes fructifères au niveau des premières sympodiales qui est compensée par la production d'un plus grand nombre total de nœuds fructifères à l'échelle de la plante : au final, on obtient plus de sympodiales et plus de nœuds par sympodiale (Hearn et al., 1979). À Okpara, le semis de juin fleurit en aoutseptembre, période régulièrement arrosée, alors que le semis retardé est en pleine floraison quand les pluies deviennent moins régulières.

En semis retardé, la production capsulaire est peu abondante et concentrée sur les deux premières positions des six premières branches fructifères. Les semis retardés réduisent les quantités de ressources disponibles pour la plante, essentiellement l'eau, mais aussi la lumière, la chaleur (sommes de températures) ou l'azote minéral issu de la matière organique du sol (Lançon et al., 2007). Les cotonniers sont ainsi limités dans leur développement (Sekloka et al., 2015) et dans leur potentiel de production aux étages supérieurs.

\subsection{Effet de la densité}

L'augmentation de la densité induit une diminution du nombre de sites fructifères par plante et du taux de rétention capsulaire. Les capsules sont moins nombreuses et concentrées sur les parties basses de la plante. En effet, les densités fortes induisent des effets accrus de compétition pour les ressources en eau et en éléments nutritifs et même pour l'interception de la lumière (Galanopoulou-Sendouka et al., 1980). Les branches végétatives $(\mathrm{BV})$ produisent peu et l'essentiel de la production est concentrée sur les toutes premières positions des 7 premières BF. Ces résultats concordent avec ceux de Jost et al. (2001) sur des cotonniers semés à des densités variant de 113000 à 405000 plantes $\cdot$ ha $^{-1}$.

La contribution des branches végétatives à la production capsulaire est généralement faible, quelle que soit la densité et tout particulièrement aux fortes densités (Jenkins et al., 1990a ; 1990b). Cette contribution est variable comme l'ont également montré Hau et al. (1987). Toutefois, pour des semis de culture pluviale réalisés à temps, les branches végétatives 
peuvent compenser une partie des chutes d'organes fructifères qui interviennent sur les sympodiales lors d'aléas climatiques (Moussa et al., 2003). Cela pourrait expliquer en partie la persistance, jusqu'à nos jours, de la culture des variétés dites végétatives dans les conditions de culture pluviale en Afrique de l'Ouest et du Centre (Lançon et al., 1990 ; Sekloka et al., 2009 ; Hougni et al., 2014).

\subsection{Variabilité génétique de la production et de la répartition capsulaire}

La répartition de la production au niveau des premiers nœuds des branches fructifères les plus basses permet de bien distinguer les variétés comparées. Les variétés plus précoces et compactes, comme Mar 88-214 et Chaco 520, présentent un pourcentage de capsules plus important dans cette partie de la plante que les autres. Jenkins et al. (1990b) ont également établi cette distinction entre cultivars dits modernes, précoces et génotypes plus anciens. On considèrera plutôt que les variétés précoces et compactes, à développement de type déterminé, sont davantage adaptées aux conditions intensives de culture tandis que les programmes de sélection conduits en Afrique ont produit des variétés plus végétatives, à forte capacité de fructification, adaptées à des conditions de culture plus risquées pour la plante (Lançon et al., 1990 ; Hau et al., 2001).

\subsection{Conclusions}

Les premières positions des branches fructifères contribuent pour plus de $60 \%$ à la production capsulaire totale et la contribution de chaque branche diminue quand son niveau d'insertion sur la tige principale augmente, comme l'ont aussi montré Kerby et al. (1981) ou Anjum et al. (2001). Ce phénomène résulterait d'une distribution préférentielle des assimilats vers les capsules plus âgées, ce qui limite le développement des capsules sur les positions plus externes des BF ainsi que sur les étages supérieurs de la plante (Jenkins et al., 1990b ; Bange et al., 2004).

Les résultats identifient les deux premières positions fructifères des huit premières sympodiales (P1-2_BF18) comme la région de concentration de la production capsulaire. Sachant que les plus grosses capsules de la plante sont produites au niveau des premières sympodiales (Hau et al., 1987 ; Constable, 1991 ; Bange et al., 2004), on peut en déduire qu'en moyenne, les huit premières branches fructifères sont les plus déterminantes pour le rendement en culture cotonnière au Bénin. Des travaux de «plant mapping », réalisés au Mississipi sur huit génotypes de différentes maturités, ont montré que les plus grandes contributions à la production de capsules et au rendement en fibre étaient apportées par les branches fructifères situées entre BF4 et BF9 (Jenkins et al., 1990a ; 1990b). Une production située plus haut sur la plante peut s'expliquer par les effets conjugués de l'irrigation associée aux fortes fertilisations azotées qui engendre un développement plus important des cotonniers et une élévation des zones de forte rétention capsulaire. Constable (1991) a constaté que les premières positions des branches fructifères situées entre les nœuds fructifères 2 à 8 (BF2 à BF8) apportaient les plus grandes contributions en nombre et en poids de capsules et, de ce fait, contribuaient le plus au rendement, ce qui est proche de nos résultats.

Une optimisation du taux de rétention au niveau des deux premières positions des huit premières branches fructifères (RP1-2_BF1-8) permettrait d'améliorer le rendement des cultures, puisqu'il s'agit de la partie de la plante où se concentre l'essentiel de la production dans nos conditions de culture. Si son héritabilité était suffisante, le critère RP1-2_BF1-8 pourrait être utilisé pour mieux apprécier le potentiel de rendement du matériel génétique en sélection. Il est toutefois très influencé par de nombreux facteurs comme la température, l'environnement cultural, les facteurs nutritionnels et physiologiques, les pressions parasitaires, les compétitions intra- et inter- branches fructifères (Reddy et al., 1992 ; Riney et al., 1997 ; Boquet et al., 2003 ; Zhao et al., 2005). Dans des conditions climatiques risquées, les itinéraires techniques favorisant une installation rapide de la production associée à une protection phytosanitaire efficace (Lançon et al., 2007 ; Rapidel et al., 2009) amélioreront le taux de rétention dans cette région de la plante et contribueront à sécuriser les rendements. Toutefois, dans les conditions normales de culture, un taux de rétention et une charge en capsules trop élevés dans les parties basses pénalisent le potentiel de production des étages supérieurs et limitent les possibilités de compensation de pertes liées à des incidents hydriques ou parasitaires en début ou en cours de culture.

\section{Bibliographie}

Anjum R., Soomro A., Chang M. \& Memon A., 2001. Effect of fruiting positions on yield in American cotton. Pak.J. Biol. Sci., 4(8), 960-962.

Azontondé A., Igué M. \& Dagbénombakin G., 2009. Carte de fertilité des sols du Bénin par zone agro-écologique $d u$ Bénin. Rapport de consultation pour le compte d'Afrique Étude, Cotonou, Bénin.

Bange M.P. \& Milroy S.P., 2004. Growth and dry matter partitioning of diverse cotton genotypes. Field Crops Res., 87(1), 73-87.

Boquet D.J. \& Moser E.B., 2003. Boll retention and boll size among intrasympodial fruiting sites in cotton. Crop Sci., 43, 195-201. 
Bourland F.M.et al., 1990. Reading the cotton plant for efficient management. In: Proceedings of the Arkansas Cotton Research Meeting, Arkansas Agricultural Experiment Station. Special Report No. 144, 45-49.

Bourland F., Oosterhuis D.M. \& Tugwell N.P., 1992. Concept for monitoring the growth and development of cotton plants using main-stem node counts. J. Prod. Agric., 5, 532538.

Burch K. \& Krieg D., 2002. The relative contribution of individual fruiting sites to cotton yield and quality. In: Proceedings of the Beltwide Cotton Conference, January 7-11, 2002, Atlanta (USA). Cordova, TN, USA: Cotton Council of America.

Constable G.A., 1991. Mapping the production and survival of fruit on field-grown cotton. Agron. J., 83, 374-378.

Cretenet M., Mohtar R.H. \& Moussa A.A., 2006. L'aléa pluviométrique dans la prise de décisions en culture cotonnière pluviale. Cah. Agric., 15(1), 109-115.

Galanopoulou-Sendouka S. et al., 1980. Effect of population density, planting date, and genotype on plant growth and development of cotton. Agron. J., 72, 347-353.

Hau B. \& Goebel S., 1987. Modifications du comportement du cotonnier en fonction de l'environnement : 2. Évolution des paramètres de productivité de neuf variétés semées à trois écartements. Coton Fibres Trop., 42(2), 117-125.

Hau B., Lançon J. \& Dessauw D., 2001. Cotton. In: Charrier A., Jacquot M., Hamon S. \& Nicolas D., eds. Tropical plant breeding. Montpellier, France: CIRAD; Enfield, NH, USA; Plymouth, UK: Science Publishers Inc., 153-176.

Hearn A. \& Room P., 1979. Analysis of crop development for cotton pest management. Prot. Ecol., 1, 265-277.

Hougni A. et al., 2014. E 956-2, K768-3, H 782-3 : trois lignées régionnalisées de cotonnier (Gossypium hirsutum L.) pour l'amélioration de la productivité de la culture cotonnière au Bénin. Atelier Scientifique INRAB (sous presse)

Jenkins J.N., McCarty J. \& Parrott W.L.Jr, 1990a. Effectiveness of fruiting sites in cotton: yield. Crop Sci., 30, 365-369.

Jenkins J.N., McCarty J. \& Parrott W.L. Jr, 1990b. Fruiting efficiency in cotton: boll size and boll set percentage. Crop Sci., 30, 857-860.

Jost P. \& Cothren J.T., 2001. Phenotypic alterations and crop maturity differences in ultra-narrow row and conventionally spaced cotton. Crop Sci., 41, 1150-1159.

Kerby T. \& Buxton D., 1981. Competition between adjacent fruiting forms in cotton. Agron. J., 73, 867-871.

Lançon J., Chanselme J.L. \& Klassou C., 1990. Bilan du progrès génétique réalisé par la recherche cotonnière au Nord Cameroun de 1960 à 1988. Coton Fibres Trop., 45(2), 145-167.

Lançon J.et al., 2007. An improved methodology for integrated crop management systems. Agron. Sustainable Dev., 27, 101-110.

Liu Z. et al., 2006. Screening for high-temperature tolerant cotton cultivars by testing in vitro pollen germination, pollen tube growth and boll retention. J. Integr. Plant Biol., 48(6), 706-714.
Moussa A.A., Cretenet M., Nibouche S. \& Gaborel C., 2003. Impact d'une attaque précoce de chenilles de la capsule sur le rendement en coton graine en fonction de la pluviosité au Nord Cameroun. In: Jamin J.Y., Seiny Boukar L. \& Floret C., eds. Actes du colloque Savanes africaines: des espaces en mutation, des acteurs face à de nouveaux défis, mai 2002, Garoua, Cameroun. N'Djamena : Prasac ; Montpellier, France : Cirad.

Nouatin G., Amouzouvi H.D. \& Dedjan J., 2014. Climate change and farmer's perception of climatic evolution in Alibori departement in Benin. Int. J. Res. Earth Environ. Sci., 1(4), 1-9.

Rapidel B. et al., 2009. Experiment-based prototyping to design and access cotton management systems in West Africa. Agron. Sustainable Dev., 29, 545-556.

Reddy K.R., Hodges H.F. \& Reddy V.R., 1992. Temperature effects on cotton fruit retention. Agron. J., 84, 26-30.

Riney J.B., Best E.C. \& Krieg D.R., 1997. Genetic and environmental factors affecting production and retention of fruiting sites. In: Proceedings of the Beltwide Cotton Conference, Memphis (USA). Cordova, TN, USA: Cotton Council of America, 1385-1387

SAS-Institute, 1988. SAS/STAT user's guide. Release 6.03 Edition. Cary, NC, USA: SAS Institute, Inc.

Sekloka E. et al., 2007a. A simple method for estimating the end of effective flowering in upland cotton (Gossypium hirsutum L.). Exp. Agric., 42(2), 163-171.

Sekloka E. et al., 2007b. Effective flowering time variations in upland cotton (Gossypium hirsutum L.) at different planting dates and stand densities in Benin. Exp.Agric., 42(2), 173 182.

Sekloka E. et al., 2008. Breeding new cotton varieties to fit the diversity of cropping conditions in Africa-Effect of plant architecture, earliness and effective flowering time on lateplanted cotton productivity. Exp. Agric., 44, 197-207.

Sekloka E. et al., 2009. I 875.3 et H 769.5, variétés prometteuses de coton (Gossypium hirsutum L.) sélectionnées au Bénin. Bull.Rech.Agron. Bénin, 63, 48-57.

Sekloka E., Lançon J., Batamoussi M. \& Thomas G., 2015. La réduction de la croissance végétative à forte densité de semis comme stratégie d'adaptation variétale aux semis tardifs en culture cotonnière pluviale au Bénin . Tropicultura, 33(4), 299-308.

Tidjani M.A. \& Akponikpè P.B.I., 2012. Évaluation des stratégies paysannes d'adaptation aux changements climatiques : cas de la production du maïs au nord Bénin. Afr. Crop Sci. J., 20(s2), 425- 441.

Tukey-Kramer C.Y., 1956. Extension of multiple range tests to group means with unequal numbers of replications. Biometrics, 12, 309-310.

Ungar E., Wallach D. \& Kletter E., 1987. Cotton response to bud and boll removal. Agron. J., 79, 491-497.

Zhao D. et al., 2005. Physiological causes of cotton fruit abscission under conditions of high temperature and enhanced ultraviolet-B radiation. Physiol. Plant., 124, 189-199.

(36 réf.) 